PROCEEDINGS OF THE

AMERICAN MATHEMATICAL SOCIETY

Volume 131, Number 9, Pages 2911-2920

S 0002-9939(03)07112-0

Article electronically published on April 9, 2003

\title{
THE MEASURE OF HOLOMORPHICNESS OF A REAL SUBMANIFOLD OF AN ALMOST HERMITIAN MANIFOLD
}

\author{
FERNANDO ETAYO
}

(Communicated by Mohan Ramachandran)

\begin{abstract}
In this note we define the measure of holomorphicness $\mu(M)$ of a compact real submanifold $M$ of an almost Hermitian manifold $(\bar{M}, \bar{J}, \bar{g})$. The number $\mu(M) \in[0,1]$ verifies the following properties: $M$ is a complex submanifold iff $\mu(M)=1$; if $\operatorname{dim} M$ is odd, then $\mu(M)=0$. Explicit examples of surfaces in $\mathbb{C}^{2}$ are obtained, showing that $\mu\left(S^{2}\right)=\frac{1}{5}$ and that $0 \leq \mu(T) \leq \frac{3}{8}$, $T$ being the Clifford torus.
\end{abstract}

\section{INTRODUCTION}

Let $M$ be a compact submanifold of an almost Hermitian manifold $(\bar{M}, \bar{J}, \bar{g})$. We are looking for a number associated to $M$ which measures the holomorphicness of the submanifold $M$. We shall obtain such a number $\mu(M) \in[0,1]$ verifying the following properties:

(a) If $\operatorname{dim}(M)$ is odd, then $\mu(M)=0$ (odd-dimensional manifolds are never holomorphic). If $M$ is a totally real submanifold, then $\mu(M)=0$.

(b) $M$ is a holomorphic submanifold of $(\bar{M}, \bar{J}, \bar{g})$ iff $\mu(M)=1$.

(c) If $\varphi:(\bar{M}, \bar{J}, \bar{g}) \rightarrow(\bar{M}, \bar{J}, \bar{g})$ is an automorphism and $M^{\prime}=\varphi(M)$, then $\mu(M)=\mu\left(M^{\prime}\right)$.

Moreover, we shall compute this number in some cases. For example, we shall consider the canonical embedding of the 2-sphere $S^{2} \subset \mathbb{C}^{2}=\left(\mathbb{R}^{4}, \bar{J}, \bar{g}\right)$ defined by $S_{0}^{2}=\left\{x_{1}^{2}+x_{2}^{2}+x_{3}^{2}=1 ; x_{4}=0\right\}$, showing that $S_{0}^{2}$ is not a generic submanifold (this fact is relevant: almost every result about real submanifolds of an almost Hermitian manifold is stated for generic submanifolds - see the definition below), and showing that $\mu\left(S_{0}^{2}\right)=\frac{1}{5}$, i.e., this sphere is, in some sense, a (20\%)-holomorphic submanifold of $\mathbb{C}^{2}$. Moreover, this is the general case: for all isometries $\varphi$ of $\left(\mathbb{R}^{4}, \bar{g}\right)$, we shall prove that the measure of holomorphicness of $\varphi\left(S_{0}^{2}\right)$ is also $\frac{1}{5}$.

On the other hand, we shall check the measure of holomorphicness of the Clifford torus $T^{\prime}=(\cos \alpha, \sin \alpha, \cos \beta, \sin \beta)$, proving $\mu\left(T^{\prime}\right)=\frac{3}{8}$ and that $0 \leq \mu\left(\varphi\left(T^{\prime}\right)\right) \leq \frac{3}{8}$, for all isometries $\varphi$ of $\left(\mathbb{R}^{4}, \bar{g}\right)$.

Received by the editors May 25, 2001.

2000 Mathematics Subject Classification. Primary 53C40; Secondary 53C55.

Key words and phrases. Measure of holomorphicness, almost Hermitian manifold, submanifold, Plücker.

The author's research was partially supported by the Spanish Ministerio de Ciencia y Technología (BFM 2002-00141). 
Plücker coordinates in the Grassmann manifold of 2-planes of $\mathbb{R}^{4}$ will play an essential role in the study of the measure of holomorphicness of compact surfaces embedded in $\mathbb{C}^{2}$.

\section{BASIC CONSTRUCTIONS}

Taking into account the almost complex structure $\bar{J}$ of $\bar{M}$ one can define some classes of submanifolds, such as:

- almost complex or holomorphic (see, e.g., [9]): $\bar{J}\left(T_{x} M\right)=T_{x} M$, for all $x \in M$;

- totally real or anti-invariant (see, e.g., [11]): $\bar{J}\left(T_{x} M\right) \subset T_{x}^{\perp} M$, for all $x \in M$;

- Cauchy-Riemann (see, e.g., [1], [2]): there exists a differentiable distribution $D: x \mapsto D_{x}$ on $M$ satisfying the following conditions: (i) $D$ is holomorphic, i.e., $\bar{J}\left(D_{x}\right)=D_{x}$, for all $x \in M$; (ii) the complementary orthogonal distribution $D^{\perp}: x \mapsto D_{x}^{\perp} \subset T_{x} M$ is anti-invariant, i.e., $\bar{J}\left(D_{x}^{\perp}\right) \subset T_{x}^{\perp} M$, for all $x \in M$;

- slant (see, e.g., (4): the angle between $\bar{J}(X)$ and $T_{x} M$ is constant for every vector $X \in T_{x} M$ and every $x \in M$. This angle is called the Wirtinger angle.

All of these are generic submanifolds, i.e., the holomorphic spaces $H_{x}=\bar{J}\left(T_{x} M\right) \cap$ $T_{x} M$ define a differentiable distribution on $M$ (see, e.g., 3]), and almost all works about submanifolds of an almost Hermitian manifold are focused on generic submanifolds of some of the above types. But one can easily find submanifolds which are not generic:

Example 1. Let $\mathbb{C}^{2}=\left(\mathbb{R}^{4}, \bar{J}, \bar{g}\right)$ be the complex plane, endowed with the complex structure given by $\bar{J}\left(\partial / \partial x^{i}\right)=\left(\partial / \partial x^{i+2}\right), \bar{J}\left(\partial / \partial x^{i+2}\right)=-\left(\partial / \partial x^{i}\right), i \in\{1,2\}$, and with the usual metric $\bar{g}=d x^{j} \otimes d x^{j}, j \in\{1, \ldots, 4\}$. The canonical embedding of the 2-sphere $S_{0}^{2} \subset \mathbb{C}^{2}$ defined by $\left\{x_{1}^{2}+x_{2}^{2}+x_{3}^{2}=1 ; x_{4}=0\right\}$ does not give a generic submanifold, because taking $p=(0,0,1,0)$ and $q=(0,1,0,0)$ one observes that $\operatorname{dim}\left(H_{p}\right)=0$ whereas $\operatorname{dim}\left(H_{q}\right)=2$.

Remark 2. Moreover, if $M$ is any surface embedded in $\mathbb{R}^{3} \subset \mathbb{R}^{4}=\mathbb{C}^{2}$ and $x \in M$, then $\bar{J}\left(T_{x} M\right)=T_{x}^{\perp} M$ (resp. $\left.\bar{J}\left(T_{x} M\right)=T_{x} M\right)$ iff $T_{x} M$ is parallel to the direction generated by $\frac{\partial}{\partial x^{2}}$ (resp. $T_{x} M$ is orthogonal to the direction generated by $\frac{\partial}{\partial x^{2}}$ ). In particular, if $M$ is compact, then there exist points in $M$ in both situations, thus proving that such a surface $M$ is a non-generic submanifold of $\mathbb{C}^{2}$. Non-generic is a more general situation than the generic one.

One can wonder what properties have the function $d: x \mapsto \operatorname{dim}\left(H_{x}\right)$ when $x \in M, M$ being a real submanifold of $(\bar{M}, \bar{J}, \bar{g})$. Obviously, $M$ being connected, the following assertions are equivalent: $d$ is continuous; $d$ is constant; $M$ is a generic submanifold. The general answer is the following:

Theorem 3 (see [7). Let $M$ be a real submanifold of an almost complex manifold $(\bar{M}, \bar{J})$. The function $d: M \rightarrow R$ given by $d(x)=\operatorname{dim}\left(H_{x}\right)$ is uppersemicontinuous, i.e., the sets $\{x \in M / d(x)<n\}$ are open subsets of $M$.

Remark 4. Taking into account the above theorem and the fact that a compact manifold cannot be immersed in $\mathbb{C}^{m}$ as a holomorphic submanifold (see [10], Corollary 1.12) one can deduce that if $M$ is an $n$-dimensional real compact submanifold 
of $\mathbb{C}^{m}$, for some $m$, then the set $\left\{x \in M / \operatorname{dim}\left(H_{x}\right)<n\right\}$ is a non-empty open subset of $M$, and thus the condition $\bar{J}\left(T_{x} M\right)=T_{x} M$ fails in more that one point $x \in M$.

Let $(\bar{M}, \bar{J}, \bar{g})$ be an almost Hermitian manifold and let $M$ be a real submanifold of $\bar{M}$. Then, the tangent space $T_{x} \bar{M}$ of $\bar{M}$ at $x \in M$ may be decomposed as the direct and normal sum $T_{x} \bar{M}=T_{x} M \oplus T_{x}^{\perp} M$, where $T_{x} M$ (resp. $T_{x}^{\perp} M$ ) is the tangent space (resp. the normal space) of $M$ at $x$. On the other hand, $\bar{J}\left(T_{x} M\right) \subset T_{x} \bar{M}$ is a vector space with $\operatorname{dim}\left(\bar{J}\left(T_{x} M\right)\right)=\operatorname{dim}\left(T_{x} M\right)$. We shall use the following projectors:

(a) $p_{1}: T_{x} \bar{M} \rightarrow T_{x} M$ and $p_{2}: T_{x} \bar{M} \rightarrow T_{x}^{\perp} M$, the orthogonal projections given by the decomposition $T_{x} \bar{M}=T_{x} M \oplus T_{x}^{\perp} M$;

(b) $\pi: T_{x} \bar{M} \rightarrow \bar{J}\left(T_{x} M\right)$, the orthogonal projection;

and the following maps:

(c) $P_{1}=\left.p_{1} \circ \bar{J}\right|_{T_{x} M}: T_{x} M \rightarrow T_{x} M$ and $P_{2}=\left.p_{2} \circ \bar{J}\right|_{T_{x} M}: T_{x} M \rightarrow T_{x}^{\perp} M$, which allows us to write $\bar{J}(X)=P_{1}(X)+P_{2}(X)$, for all $X \in T_{x} M$;

(d) $Q_{x}=P_{1} \circ P_{1}: T_{x} M \rightarrow T_{x} M$;

(e) $F_{x}=\left.p_{1} \circ \pi\right|_{T_{x} M}: T_{x} M \rightarrow T_{x} M$.

The maps defined in (c) and (d) have been introduced by Chen in [4], where some properties are also proved. We summarize them together with others in the following lemma. Observe that $M$ inherits from $(\bar{M}, \bar{J}, \bar{g})$ the Riemannian metric $g=\left.\bar{g}\right|_{M}$, and, if $M$ is holomorphic, also the almost complex structure, but, in any case one can consider the tangent component of $\bar{J}$, which is the map $P_{1}$.

Lemma 5. Using the above notation, one has:

(1) $\bar{g}\left(P_{1}(X), Y\right)+\bar{g}\left(X, P_{1}(Y)\right)=0$ and then $Q$ is self-adjoint: $\bar{g}\left(Q_{x}(X), Y\right)=$ $\bar{g}\left(X, Q_{x}(Y)\right)$. Therefore $T_{x} M$ may be decomposed as an orthogonal direct sum of eigenspaces of $Q_{x}$, i.e., $T_{x} M=\mathcal{D}_{x}^{1} \oplus \ldots \oplus \mathcal{D}_{x}^{k(x)}$. Each eigenspace $\mathcal{D}_{x}^{i}$, associated to the eigenvalue $\lambda_{i}$, is $P_{1}$-invariant and is even-dimensional if $\lambda_{i} \neq 0$. Finally, each eigenvalue $\lambda_{i} \in[-1,0]$.

(2) $F_{x}=-Q_{x}$, and then $\lambda$ is an eigenvalue of $F_{x}$ iff $-\lambda$ is an eigenvalue of $Q_{x}$. In particular, if $\operatorname{dim}(M)$ is odd, then 0 is an eigenvalue of $F_{x}$.

(3) The eigenspace of $F_{x}$ associated to the eigenvalue 1 coincides with $H_{x}=$ $\bar{J}\left(T_{x} M\right) \cap T_{x} M$.

The spectrum of $F_{x}$, i.e., the set of eigenvalues of $F_{x}$, will be denoted by $\operatorname{Spec}\left(F_{x}\right)$, for all $x \in M$. If $\operatorname{dim}(M)=n$ and $x \in M$, then, by the above lemma, one has $\operatorname{Spec}\left(F_{x}\right)=\left\{\lambda_{1}, \lambda_{1}, \ldots, \lambda_{k}, \lambda_{k}, 0, \ldots, 0\right\}$, with $2 k \leq n, \lambda_{i} \neq 0\left(\lambda_{i}=\lambda_{j}\right.$ is possible, with $i \neq j)$, and $\operatorname{det}\left(F_{x}\right) \in[0,1]$. If $\operatorname{dim}(M)$ is odd, then $\operatorname{det}(F) \equiv 0$.

Remark 6. Some classes of submanifolds shown in the above section can be characterized by their spectra. If $\operatorname{dim}(M)=n$, one has:

(a) $M$ is an almost complex submanifold iff $\operatorname{Spec}\left(F_{x}\right)=\{1, \ldots, 1\}$, for all $x \in M$;

(b) $M$ is a totally real submanifold iff $\operatorname{Spec}\left(F_{x}\right)=\{0, \ldots, 0\}$, for all $x \in M$;

(c) $M$ is a CR-submanifold iff $\operatorname{Spec}\left(F_{x}\right)=\left\{1, .^{2 k}, 1,0, \ldots 0\right\}$, for all $x \in M$;

(d) $M$ is a slant submanifold iff $\operatorname{Spec}\left(F_{x}\right)=\left\{\cos ^{2} \vartheta, \ldots, \cos ^{2} \vartheta\right\}$ (as one can show as a consequence of Proposition 8);

(e) $M$ is a generic submanifold iff $\operatorname{Spec}\left(F_{x}\right)=\left\{1,2 k, 1, \lambda_{2 k+1}, \ldots, \lambda_{n}\right\}$, with $\lambda_{2 k+i}$ $\neq 1$, for all $x \in M$. 
Now, we shall recall a class of real submanifolds of an almost Hermitian manifold, introduced by us in [6], which contains examples of both generic and non-generic submanifolds:

Definition 7. A submanifold $M$ of an almost Hermitian manifold $(\bar{M}, \bar{J}, \bar{g})$ is said to be quasi-slant if, for each $x \in M$, the angle $\vartheta(X)$ between $\bar{J}(X)$ and $T_{x} M$ is a constant, for all non-zero vectors $X \in T_{x} M$, i.e., it does not depend on the choice of $X \in T_{x} M$, but it depends on the choice of the point $x \in M$. Then, the angle function can be defined on the submanifold $M$ and it will be denoted by $\vartheta: M \rightarrow\left[0, \frac{\pi}{2}\right], \vartheta(x)=\vartheta_{x}$.

Proposition 8 (see [6], Theorem 2.3). Let $M$ be a submanifold of an almost Hermitian manifold $(\bar{M}, \bar{J}, \bar{g})$. Then, $M$ is a quasi-slant submanifold iff there exists a function $f$ on $M$ with values in $[0,1]$, such that $\operatorname{Spec}\left(F_{x}\right)=\{f(x), \ldots, f(x)\}$. In this case, $f(x)=\cos ^{2} \vartheta_{x}, \forall x \in M$.

Taking into account the above proposition and Lemma 5(1) one has:

Corollary 9. A surface $M$ embedded in an almost Hermitian manifold $(\bar{M}, \bar{J}, \bar{g})$ is always a quasi-slant submanifold.

\section{Measure of holomorphicness}

Let $M$ be a compact submanifold of an almost Hermitian manifold $(\bar{M}, \bar{J}, \bar{g})$. We shall define the number $\mu(M)$ :

Definition 10. Let $M$ be a compact submanifold of an almost Hermitian manifold $(\bar{M}, \bar{J}, \bar{g})$ and let $\omega$ be the volume element associated to the metric $g=\left.\bar{g}\right|_{M}$ on $M$. Then

$$
\mu(M)=\frac{\int_{M} \operatorname{det}(F) \omega}{\operatorname{vol}(M)},
$$

where $\operatorname{vol}(M)$ denotes the Riemannian volume of $(M, g)$.

Obviously, one has $0 \leq \mu(M) \leq 1$. On the other hand, taking into account Lemma 5 (2) one can check that if $\operatorname{dim}(M)$ is odd, then $\mu(M)=0$. If $M$ is totally real, $\mu(M)=0$, by Remark 6(b). An easy exercise proves that if $\varphi$ is an automorphism of $(\bar{M}, \bar{J}, \bar{g})$, i.e., if $\varphi$ is an isometry verifying $\varphi_{*} \circ \bar{J}=\bar{J} \circ \varphi_{*}$, then $\operatorname{Spec}\left(F_{x}\right)$ at $x \in M$ and $\operatorname{Spec}\left(F_{\varphi(x)}^{\prime}\right)$ at $\varphi(x) \in M^{\prime}$ coincide $\left(F\right.$ and $F^{\prime}$ denoting the endomorphisms of $M$ and $\left.M^{\prime}\right)$, thus showing that $\mu(M)=\mu\left(M^{\prime}\right)$. Finally, the desired property (b) can be obtained as a consequence of Theorem 3 ,

Proposition 11. Let $M$ be a compact submanifold of an almost Hermitian manifold $(\bar{M}, \bar{J}, \bar{g})$. Then, $M$ is a holomorphic submanifold of $(\bar{M}, \bar{J}, \bar{g})$ iff $\mu(M)=1$.

Proof. The implication $\Rightarrow$ is trivial, by Remark 6(a). On the other hand, let us assume that $M$ is not a holomorphic submanifold of $(\bar{M}, \bar{J}, \bar{g})$. Then Theorem 3 proves that the set $A=\{x \in M / d(x)<\operatorname{dim}(M)\}$ is a non-empty open subset of $M$, and, by Lemma $5(3)$, one has that $\operatorname{det}\left(F_{x}\right)<1$, for all $x \in A$, thus showing that $\int_{M} \operatorname{det}(F) \omega<\operatorname{vol}(M)$, and then $\mu(M)<1$.

In order to obtain some explicit examples of the calculus of $\mu(M)$, when $M$ is a surface immersed in $\mathbb{C}^{2}=\left(\mathbb{R}^{4}, \bar{J}, \bar{g}\right)$, we shall need a useful way of checking the Wirtinger angle of the tangent plane of $M$ at any point. This will be made by means of Plücker coordinates in the Grassmann manifold $G(2,4)$ of vector planes in 
$\mathbb{R}^{4}$. One can immerse $G(2,4)$ in $P_{5}(\mathbb{R})$ by using Plücker coordinates: if the vectors $a=\left(a_{1}, a_{2}, a_{3}, a_{4}\right)$ and $b=\left(b_{1}, b_{2}, b_{3}, b_{4}\right)$ define a basis of the plane $\Pi$, then one can define the numbers $p_{i j}=\left|\begin{array}{ll}a_{i} & a_{j} \\ b_{i} & b_{j}\end{array}\right|$, with $1 \leq i<j \leq 4$, and one can associate to the plane $\Pi$ the homogeneous coordinates $\left(p_{12}: p_{13}: p_{14}: p_{23}: p_{24}: p_{34}\right) \in P_{5}(\mathbb{R})$. As is well known, under a change of the basis in the plane $\Pi$, the Plücker coordinates are multiplied by the determinant of the transformation matrix and $G(2,4)$ can be identified with the quadric $\left\{p_{12} p_{34}-p_{13} p_{24}+p_{14} p_{23}=0\right\} \subset P_{5}(\mathbb{R})$. The complex structure $\bar{J}$ in $\mathbb{R}^{4}$ is given by $\bar{J}\left(a_{1}, a_{2}, a_{3}, a_{4}\right)=\left(-a_{3},-a_{4}, a_{1}, a_{2}\right)$.

We can obtain the expression of the function $W: G(2,4) \rightarrow \mathbb{R}$, which maps each plane $\Pi$ to $\cos \vartheta, \vartheta$ being the Wirtinger angle:

Proposition 12. Let $\Pi \in G(2,4)$ and let $\left\{a=\left(a_{1}, a_{2}, a_{3}, a_{4}\right), b=\left(b_{1}, b_{2}, b_{3}, b_{4}\right)\right\}$ be an orthonormal basis of $\Pi$. Then,

$$
W(\Pi)=W\left(p_{12}: p_{13}: p_{14}: p_{23}: p_{24}: p_{34}\right)=\left|p_{13}+p_{24}\right| .
$$

In particular, if $\Pi$ is a complex (resp. totally real) submanifold of $\mathbb{C}^{2}$, then $\mid p_{13}+$ $p_{24} \mid=1($ resp. $=0)$.

Proof. As $P_{1}(a) \in \Pi$, one can write $P_{1}(a)=\alpha a+\beta b$. Taking into account that $a \perp b, \bar{J}(a) \perp a$ and $\left(\bar{J}(a)-P_{1}(a)\right) \perp a$ one can prove that $\alpha=0$. Then, $\vartheta=\angle(\bar{J}(a), \Pi)=\angle\left(\bar{J}(a), P_{1}(a)\right)=\angle(\bar{J}(a), b)+\varepsilon \pi$, with $\varepsilon=0$ (resp. $\left.\varepsilon=1\right)$ if $\beta>0$ (resp. $\beta<0)$. On the other hand, $\cos \angle(\bar{J}(a), b)=\bar{J}(a) \cdot b=\left(-a_{3},-a_{4}, a_{1}, a_{2}\right)$. $\left(b_{1}, b_{2}, b_{3}, b_{4}\right)=p_{13}+p_{24}$, thus proving the result.

Then, taking into account Proposition 8 , Corollary 9 and the above Proposition 12 we obtain the following result which will allow us to check the measure of holomorphicness of a surface in $\mathbb{C}^{2}$.

Corollary 13. If $M$ is a compact surface $M \subset \mathbb{C}^{2}$, then $\mu(M)=\frac{\int_{M}\left(p_{13}+p_{24}\right)^{4} \omega}{\operatorname{vol}(M)}$, where $p_{13}$ and $p_{24}$ are the correspondent Plücker coordinates of the tangent planes to $M$.

We shall end this section with the following

Remark 14. If $M$ is a compact surface $M \subset \mathbb{C}^{2}$ with $\mu(M)=0$, then $M$ is a totally real submanifold of $\mathbb{C}^{2}$ : if there exists $x \in M$ such that $\vartheta_{x} \neq \frac{\pi}{2}$, then there exists a neighbourhood of $x$ verifying $\vartheta \neq \frac{\pi}{2}$, and thus $\int_{M} \operatorname{det}(F) \omega>0$.

\section{EXAmples}

We shall obtain explicit calculus for the measure of holomorphicness of surfaces in $\mathbb{C}^{2}$ : We shall begin by checking the number $\mu\left(S_{0}^{2}\right)$ for the embedding $S_{0}^{2} \subset \mathbb{R}^{3} \subset \mathbb{C}^{2}$ defined in Example 1.

Example 15. Let $\mathbb{C}^{2}=\left(\mathbb{R}^{4}, \bar{J}, \bar{g}\right)$ be the complex plane endowed with the Kähler structure given in Example 1. We consider the embedding of the 2-sphere $S_{0}^{2} \subset$ $\mathbb{R}^{4}=\mathbb{C}^{2}$ defined by $\left\{x_{1}^{2}+x_{2}^{2}+x_{3}^{2}=1 ; x_{4}=0\right\}$. Let us recall that the points $(0, \pm 1,0,0) \in S_{0}^{2}$ are the "complex points" (i.e., their tangent planes are $\bar{J}$-invariant), whereas the points of the circle $\left\{x_{2}=0\right\} \cap S_{0}^{2}$ are the "totally real ones" (in the sense that their tangent planes are totally real). 
If $q=\left(q_{1}, q_{2}, q_{3}, 0\right) \in S_{0}^{2}$, with $q \neq(0, \pm 1,0,0)$, then $\{v, w\}$ is an orthonormal basis of the tangent plane $T_{q} S_{0}^{2}$, where

$$
v=\left(\frac{q_{1} q_{2}}{\sqrt{q_{1}^{2}+q_{3}^{2}}}, \frac{-q_{1}^{2}-q_{3}^{2}}{\sqrt{q_{1}^{2}+q_{3}^{2}}}, \frac{q_{2} q_{3}}{\sqrt{q_{1}^{2}+q_{3}^{2}}}, 0\right), \quad w=\left(\frac{-q_{3}}{\sqrt{q_{1}^{2}+q_{3}^{2}}}, 0, \frac{q_{1}}{\sqrt{q_{1}^{2}+q_{3}^{2}}}, 0\right) .
$$

Then, we can compute the Wirtinger angle of the plane $T_{q} S_{0}^{2}$ taking into account Proposition 12: $\cos \vartheta=\left|p_{13}+p_{24}\right|=\left|\frac{q_{1}^{2} q_{2}+q_{2} q_{3}^{2}}{q_{1}^{2}+q_{3}^{2}}+0\right|=\left|q_{2}\right|$, thus showing that, for each $\vartheta \in\left(0, \frac{\pi}{2}\right]$, the points whose tangent plane have Wirtinger angle $\vartheta$ define a pair of circles $S_{0}^{2} \cap\left\{\left|x_{2}\right|=\cos \vartheta\right\}$.

Finally, we can compute $\mu\left(S_{0}^{2}\right)$. In this case, taking into account Proposition 8 the function $\operatorname{det}(F): S_{0}^{2} \rightarrow \mathbb{R}$ is given by $\operatorname{det}(F)\left(x_{1}, x_{2}, x_{3}, 0\right)=x_{2}^{4}$. We choose the following spherical coordinates for $S_{0}^{2}$ :

$$
x_{1}=\cos v \cos u ; x_{2}=\sin v ; x_{3}=\cos v \sin u ; u \in[0,2 \pi), v \in\left[-\frac{\pi}{2}, \frac{\pi}{2}\right] .
$$

Then the differential of area is $\sqrt{\operatorname{det} g} d u d v=\cos v d u d v$, thus leading us to: $\int_{S_{0}^{2}} \operatorname{det}(F)=\int_{u=0}^{2 \pi} \int_{v=-\frac{\pi}{2}}^{\frac{\pi}{2}} \sin ^{4} v \cos v d u d v=\frac{4 \pi}{5}$, and one can conclude that $\mu\left(S_{0}^{2}\right)=\frac{4 \pi / 5}{4 \pi}=\frac{1}{5}$.

We shall show two different Clifford flat torus:

Example 16. (a) If one considers the torus

$$
T=\{f(\alpha, \beta)=(\cos \alpha, \cos \beta, \sin \alpha, \sin \beta)\} \subset \mathbb{C}^{2}
$$

one easily checks that the partial derivatives $f_{\alpha}, f_{\beta}$ define an orthonormal basis of the tangent plane at any point of $T$, verifying $\left|p_{13}+p_{24}\right|=0$, thus showing that $T$ is a totally real compact surface in $\mathbb{C}^{2}$.

(b) On the other hand, let us consider the torus

$$
T^{\prime}=\{f(\alpha, \beta)=(\cos \alpha, \sin \alpha, \cos \beta, \sin \beta)\} \subset \mathbb{C}^{2} .
$$

Then, $f_{\alpha}$ and $f_{\beta}$ at $q \in T^{\prime}$ also define an orthonormal basis of $T_{q} T^{\prime}$, and the Wirtinger angle $\vartheta$ of this tangent plane is given by: $\cos \vartheta=\left|p_{13}+p_{24}\right|=\sin \alpha \sin \beta+$ $\cos \alpha \cos \beta$, and then

$$
\mu\left(T^{\prime}\right)=\frac{1}{4 \pi^{2}} \int_{\alpha=0}^{2 \pi} \int_{\beta=0}^{2 \pi}(\sin \alpha \sin \beta+\cos \alpha \cos \beta)^{4} d \alpha d \beta=\frac{1}{4 \pi^{2}} \frac{3 \pi^{2}}{2}=\frac{3}{8} .
$$

Observe that there exists a global isometry $\varphi:\left(\mathbb{R}^{4}, \bar{g}\right) \rightarrow\left(\mathbb{R}^{4}, \bar{g}\right)$ such that $T^{\prime}=\varphi(T)$.

\section{The Measure of holomorphiCnESS OF DifFERENT ISOMETRIC EMBEDDINGS OF A COMPACT SURFACE INTO $\mathbb{C}^{2}$}

Let $M_{0}$ be a compact 2-dimensional submanifold of the Kähler manifold $\mathbb{C}^{2}=$ $\left(\mathbb{R}^{4}, \bar{J}, \bar{g}\right)$. We want to study the values $\mu\left(\varphi\left(M_{0}\right)\right)$, when $\varphi:\left(\mathbb{R}^{4}, \bar{g}\right) \rightarrow\left(\mathbb{R}^{4}, \bar{g}\right)$ is an isometry. If $\varphi$ is a translation, then $\mu\left(\varphi\left(M_{0}\right)\right)=\mu\left(M_{0}\right)$. Then, we can assume that $\varphi \in O(4)$, and then we can define a bijection between the set

$$
\left\{\varphi\left(M_{0}\right), \varphi \text { isometry of }\left(\mathbb{R}^{4}, \bar{g}\right)\right\}
$$

and the Lie group of orthogonal matrices $O(4)$. This bijection allows us to define the function $\mu: O(4) \rightarrow[0,1]$ given by $\mu(\varphi)=\mu\left(\varphi\left(M_{0}\right)\right)$. As $O(4)$ is a compact manifold the maximum and the minimum of $\mu$ are reached. Taking into account 
that a compact manifold cannot be immersed in $\mathbb{C}^{m}$ as a holomorphic submanifold (see 10], Corollary 1.12), one has $\mu(\varphi)<1$, for all $\varphi \in O(4)$, and one can associate to $M_{0}$ the numbers $\alpha\left(M_{0}\right)=\min \left(\mu\left(\varphi\left(M_{0}\right)\right)\right)$ and $\beta\left(M_{0}\right)=\max \left(\mu\left(\varphi\left(M_{0}\right)\right)\right)$, corresponding to the "less" and "more" holomorphic immersions of $\left(M_{0}, g=\left.\bar{g}\right|_{M}\right)$.

Thus, for each compact surface one has $0 \leq \alpha\left(M_{0}\right) \leq \mu\left(\varphi\left(M_{0}\right)\right) \leq \beta\left(M_{0}\right)<1$, for all $\varphi \in O(4)$. It is easily shown that for all $\varepsilon>0$ one can find a compact surface $M_{0}$ such that $\beta\left(M_{0}\right)>1-\varepsilon$.

Let us recall the above two examples. In the case of the sphere, we have obtained $\mu\left(S_{0}^{2}\right)=\frac{1}{5}$. This is the situation for all the spheres $\varphi\left(S_{0}^{2}\right)$, where $\varphi$ is an isometry of $\left(\mathbb{R}^{4}, \bar{g}\right)$, as we shall see in the following:

Proposition 17. Let $S_{0}^{2} \subset \mathbb{C}^{2}$ be the sphere given in Example 15. Then $\mu\left(\varphi\left(S_{0}^{2}\right)\right)=$ $\frac{1}{5}$, for all isometries $\varphi$ of $\left(\mathbb{R}^{4}, \bar{g}\right)$, i.e., $\alpha\left(S_{0}^{2}\right)=\beta\left(S_{0}^{2}\right)=\frac{1}{5}$.

Proof. The isometry $\varphi$ moves the hyperplane $\left\{x_{4}=0\right\}$ onto another hyperplane $H$, through the origin, and then $\varphi\left(S_{0}^{2}\right)=S^{3} \cap H$, where $S^{3}$ is the unit sphere in $\mathbb{R}^{4}$. Taking into account that any hyperplane can be decomposed as an orthogonal direct sum of a $\bar{J}$-invariant plane and a line, one can easily prove that there exists an automorphism $\psi$ of $\left(\mathbb{R}^{4}, \bar{J}, \bar{g}\right)$ carrying the hyperplane $\left\{x_{4}=0\right\}$ onto $H$, and then carrying $S^{3} \cap\left\{x_{4}=0\right\}$ onto $S^{3} \cap H$, i.e., the sphere $S_{0}^{2}$ onto $\varphi\left(S_{0}^{2}\right)$, thus proving $\mu\left(\varphi\left(S_{0}^{2}\right)\right)=\mu\left(\psi\left(S_{0}^{2}\right)\right)=\mu\left(S_{0}^{2}\right)=\frac{1}{5}$.

As is well known, there are no slant immersions of the sphere $S^{2}$ into $\mathbb{C}^{2}$ with $\vartheta \in\left[0, \frac{\pi}{2}\right)$ (cf. [5]). Now we shall prove that a compact surface embedded in a real hyperplane of $\mathbb{C}^{2}$ is not a totally real submanifold.

Proposition 18. Let $M$ be a compact surface embedded in a real hyperplane $H$ of $\mathbb{C}^{2}$. Then, $\mu(M)>0$.

Proof. If $H=\left\{x_{4}=0\right\}$, then the result is obvious by Remark 2 and then $\mu(M)>$ 0 . Then, for all hyperplanes $H$ one can consider an automorphism $\psi$ of $\left(\mathbb{R}^{4}, \bar{J}, \bar{g}\right)$ carrying the hyperplane $H$ onto $\left\{x_{4}=0\right\}$ and then $\mu(M)=\mu(\psi(M))>0$.

In the case of the Clifford torus we have obtained two isometric immersions $T$ and $T^{\prime}$ (see Example 16) with $\mu(T)=0$ and $\mu\left(T^{\prime}\right)=\frac{3}{8}$. We shall prove that these are the minimum and the maximum values. First of all, we shall need the following results:

Lemma 19. If $\{a, b\}$ is an orthonormal basis of a plane $\Pi$ and $p_{i j}=\left|\begin{array}{cc}a_{i} & a_{j} \\ b_{i} & b_{j}\end{array}\right|$, with $1 \leq i<j \leq 4$, then $\sum_{i<j}\left(p_{i j}\right)^{2}=1$.

Proof. Let $a=\left(a_{1}, a_{2}, a_{3}, a_{4}\right)$ and $b=\left(b_{1}, b_{2}, b_{3}, b_{4}\right)$. Then

$$
\begin{aligned}
\sum_{i<j}\left(p_{i j}\right)^{2}= & \left(a_{1}^{2} b_{2}^{2}+a_{2}^{2} b_{1}^{2}-2 a_{1} a_{2} b_{1} b_{2}\right)+\left(a_{1}^{2} b_{3}^{2}+a_{3}^{2} b_{1}^{2}-2 a_{1} a_{3} b_{1} b_{3}\right) \\
& +\left(a_{1}^{2} b_{4}^{2}+a_{4}^{2} b_{1}^{2}-2 a_{1} a_{4} b_{1} b_{4}\right)+\left(a_{2}^{2} b_{3}^{2}+a_{3}^{2} b_{2}^{2}-2 a_{2} a_{3} b_{2} b_{3}\right) \\
& +\left(a_{2}^{2} b_{4}^{2}+a_{4}^{2} b_{2}^{2}-2 a_{2} a_{4} b_{2} b_{4}\right)+\left(a_{3}^{2} b_{4}^{2}+a_{4}^{2} b_{3}^{2}-2 a_{3} a_{4} b_{3} b_{4}\right) \\
= & a_{1}^{2}\left(1-b_{1}^{2}\right)+a_{2}^{2}\left(1-b_{2}^{2}\right)+a_{3}^{2}\left(1-b_{3}^{2}\right)+a_{4}^{2}\left(1-b_{4}^{2}\right) \\
& -2\left(a_{1} a_{2} b_{1} b_{2}+a_{1} a_{3} b_{1} b_{3}+a_{1} a_{4} b_{1} b_{4}+a_{2} a_{3} b_{2} b_{3}+a_{2} a_{4} b_{2} b_{4}+a_{3} a_{4} b_{3} b_{4}\right) \\
= & 1-\left(a_{1} b_{1}+a_{2} b_{2}+a_{3} b_{3}+a_{4} b_{4}\right)^{2}=1 .
\end{aligned}
$$


Lemma 20. Let $\Pi \in G(2,4)$ be a plane with Plücker coordinates $\left(q_{12}: q_{13}\right.$ : $\left.q_{14}: q_{23}: q_{24}: q_{34}\right)$. Then, the orthogonal plane $\Pi^{\perp}$ has Plücker coordinates $\left(q_{34}:-q_{24}: q_{23}: q_{14}:-q_{13}: q_{12}\right)$.

Proof. Two planes $\Pi$ and $\Pi^{\prime}$ in $\mathbb{R}^{4}$ are orthogonal iff the real projective lines $P(\Pi)$ and $P\left(\Pi^{\prime}\right)$ are dual in $P\left(\mathbb{R}^{4}\right)=P_{3}(\mathbb{R})$. As is well known the Plücker coordinates of $\Pi$ coincide with those of $P(\Pi)$. The Plücker coordinates $\left(r_{12}: r_{13}: r_{14}: r_{23}: r_{24}\right.$ : $r_{34}$ ) of the dual space $P\left(\Pi^{\prime}\right)$ of $P(\Pi)$ satisfy $r_{i_{1} i_{2}}=\varepsilon_{i_{1} i_{2} i_{3} i_{4}} q_{i_{3} i_{4}}$ (cf. [8], pp. 294 and 297), where $\varepsilon_{i_{1} i_{2} i_{3} i_{4}}$ is the sign of the permutation.

Then we can obtain:

Proposition 21. Let $T \subset \mathbb{C}^{2}$ be the Clifford torus given in Example 16)(a). If $\varphi$ is an isometry of $\left(\mathbb{R}^{4}, \bar{g}\right)$, then $0 \leq \mu(\varphi(T)) \leq \frac{3}{8}$.

Proof. Let $A \in O(4)$ be the matrix of $\varphi$. As the partial derivatives $f_{\alpha}, f_{\beta}$ define an orthonormal basis of a tangent plane at any point $x$ of $T$, then $v=A f_{\alpha}=$ $\left(v_{1}, v_{2}, v_{3}, v_{4}\right)$ and $w=A f_{\beta}=\left(w_{1}, w_{2}, w_{3}, w_{4}\right)$ define an orthonormal basis of the tangent plane at the point $\varphi(x)$ of $\varphi(T)$. Let

$$
p_{13}=\left|\begin{array}{cc}
v_{1} & v_{3} \\
w_{1} & w_{3}
\end{array}\right| \quad \text { and } \quad p_{24}=\left|\begin{array}{cc}
v_{2} & v_{4} \\
w_{2} & w_{4}
\end{array}\right| \text {. }
$$

We have to compute the maximum and the minimum of

$$
\mu(\varphi(T))=\int_{\alpha=0}^{2 \pi} \int_{\beta=0}^{2 \pi}\left(p_{13}+p_{24}\right)^{4} d \alpha d \beta,
$$

when $\varphi$ is an isometry of $\left(\mathbb{R}^{4}, \bar{g}\right)$.

Let $A=\left(a_{i j}\right)$ with $1 \leq i, j \leq 4$ be the matrix of $\varphi$. A direct calculation shows that

$$
\begin{aligned}
p_{13}= & \left(a_{11} a_{32}-a_{31} a_{12}\right) \sin \alpha \sin \beta+\left(a_{31} a_{14}-a_{11} a_{34}\right) \sin \alpha \cos \beta \\
& +\left(a_{33} a_{12}-a_{13} a_{32}\right) \cos \alpha \sin \beta+\left(a_{13} a_{34}-a_{33} a_{14}\right) \cos \alpha \cos \beta \\
= & q_{12} \sin \alpha \sin \beta-q_{14} \sin \alpha \cos \beta+q_{23} \cos \alpha \sin \beta+q_{34} \cos \alpha \cos \beta,
\end{aligned}
$$

where $q_{i j}$ are the Plücker coordinates of the plane generated by the first and third row vectors of $A$, i.e., by the vectors $a_{1}=\left(a_{11}, a_{12}, a_{13}, a_{14}\right)$ and $a_{3}=$ $\left(a_{31}, a_{32}, a_{33}, a_{34}\right)$.

A similar argument shows that

$$
p_{24}=r_{12} \sin \alpha \sin \beta-r_{14} \sin \alpha \cos \beta+r_{23} \cos \alpha \sin \beta+r_{34} \cos \alpha \cos \beta,
$$

where $r_{i j}$ are the Plücker coordinates of the plane generated by the second and fourth row vectors of $A$, i.e., by the vectors $a_{2}=\left(a_{21}, a_{22}, a_{23}, a_{24}\right)$ and $a_{4}=$ $\left(a_{41}, a_{42}, a_{43}, a_{44}\right)$.

Then, we have

$$
\begin{aligned}
p_{13}+p_{24}= & \left(q_{12}+r_{12}\right) \sin \alpha \sin \beta-\left(q_{14}+r_{14}\right) \sin \alpha \cos \beta \\
& +\left(q_{23}+r_{23}\right) \cos \alpha \sin \beta+\left(q_{34}+r_{34}\right) \cos \alpha \cos \beta .
\end{aligned}
$$

Now, taking into account that $A$ is the matrix of an isometry $\varphi$ we can consider the row vectors of $A$ as an orthonormal basis $\left\{a_{1}, a_{2}, a_{3}, a_{4}\right\}$ of $\left(\mathbb{R}^{4}, \bar{g}\right)$ and then the planes generated by $\left\{a_{1}, a_{3}\right\}$ and $\left\{a_{2}, a_{4}\right\}$ are orthogonal. By the above Lemma 20 we have:

$$
p_{13}+p_{24}=a \sin \alpha \sin \beta-b \sin \alpha \cos \beta+b \cos \alpha \sin \beta+a \cos \alpha \cos \beta,
$$


where $a=q_{12}+q_{34}$ and $b=q_{14}+q_{23}$. Let us check $\mu(\varphi(T))$ by using the expression obtained in Corollary 13:

$$
\begin{aligned}
& \mu(\varphi(T))=\frac{1}{4 \pi^{2}} \int_{\alpha=0}^{2 \pi} \int_{\beta=0}^{2 \pi}\left(p_{13}+p_{24}\right)^{4} d \alpha d \beta \\
& =\frac{1}{4 \pi^{2}} \int_{\alpha=0}^{2 \pi} \int_{\beta=0}^{2 \pi}(a \sin \alpha \sin \beta-b \sin \alpha \cos \beta+b \cos \alpha \sin \beta+a \cos \alpha \cos \beta)^{4} d \alpha d \beta \\
& =\frac{1}{4 \pi^{2}} \frac{3 \pi^{2}}{2}\left(a^{2}+b^{2}\right)^{2}=\frac{3}{8}\left(a^{2}+b^{2}\right)^{2} .
\end{aligned}
$$

When $A=i d$, then $a=b=0$ and we have the minimum. When

$$
A=\left(\begin{array}{llll}
1 & 0 & 0 & 0 \\
0 & 0 & 1 & 0 \\
0 & 1 & 0 & 0 \\
0 & 0 & 0 & 1
\end{array}\right),
$$

we have $a^{2}+b^{2}=1$, and then $\varphi(T)=T^{\prime}$. We shall prove that this is the upper bound.

Let us assume that $a^{2}+b^{2}>1$. Then, developping the expressions of $a$ and $b$ one obtains:

$$
a^{2}+b^{2}>1 \Leftrightarrow\left(q_{12}\right)^{2}+\left(q_{34}\right)^{2}+\left(q_{14}\right)^{2}+\left(q_{23}\right)^{2}+2\left(q_{12} q_{34}+q_{14} q_{23}\right)>1
$$

and by Lemma 19 this is equivalent to

$$
1-\left(q_{13}\right)^{2}-\left(q_{24}\right)^{2}+2\left(q_{12} q_{34}+q_{14} q_{23}\right)>1,
$$

and, finally, as $\left(q_{i j}\right)$ are Plücker coordinates verifying $q_{12} q_{34}-q_{13} q_{24}+q_{14} q_{23}=0$, we obtain

$$
0>\left(q_{13}-q_{24}\right)^{2},
$$

which is not possible, thus finishing the proof.

\section{REFERENCES}

[1] Bejancu, A., CR-submanifolds of a Kaehler manifold I. Proc. Amer. Math. Soc. 69 (1978), 134-142. MR 57:7486

[2] Bejancu, A., Geometry of CR-Submanifolds. D. Reidel, Dordrecht, 1986. MR 87k:53126

[3] Chen, B. Y., Geometry of Submanifolds and its Applications. Sci. Univ. Tokyo, 1981. MR 82m:53051

[4] Chen, B. Y., Slant immersions. Bull. Austral. Math. Soc. 41 (1990), 135-147. MR 91h:53051

[5] Chen, B. Y., Tazawa, Y., Slant submanifolds in complex Euclidean spaces. Tokyo J. Math. 14 (1991), 101-120. MR 92e:53075

[6] Etayo, F., On quasi-slant submanifolds of an almost Hermitian manifold. Publ. Math. Debrecen 53 (1998), 217-223. MR 99j:53073

[7] Etayo, F., Fioravanti, M., Trías, U. R., The dimension function of holomorphic spaces of a real submanifold of an almost complex manifold. Czechoslovak Math. J. 51 (126) (2001), 139-141. MR 2002a:53037

[8] Hodge, W. V. D., Pedoe, D., Methods of Algebraic Geometry, I. Cambridge Univ. Press, Cambridge, 1994 (first published in 1947 MR 10:396b). MR 95d:14002a

[9] Oguie, K., Differential Geometry of Kähler Submanifolds. Advances in Math. 13 (1974), 73-114. MR 49:11444 
[10] Wells, R. O. Jr., Differential Analysis on Complex Manifolds. Springer, New York, 1980. MR 83f:58001

[11] Yano, K., Kon, M., Anti-invariant Submanifolds. M. Dekker Inc., New York, 1976. MR 54:13799

Departamento de Matemáticas, Estadística y Computación, Facultad de Ciencias, Universidad de Cantabria, Avda. de los Castros, s.n., E-39071 Santander, Spain

E-mail address: etayof@unican.es 